\title{
Effectiveness of a 23-valent Pneumococcal Polysaccharide Vaccine for the Prevention of Pneumococcal Pneumonia in the Elderly with Chronic Respiratory Diseases: A Case-control Study of a Single Center
}

Toshihiro Masuda

Shizuoka General Hospital

Eiji Nakatani ( $\square$ nakatani.eiji.int@gmail.com )

Shizuoka General Hospital

Toshihiro Shirai

Shizuoka General Hospital

Taisuke Akamatsu

Shizuoka General Hospital

Kanami Tamura

Shizuoka General Hospital

Shingo takahashi

Shizuoka General Hospital

Yuko Tanaka

Shizuoka General Hospital

Hirofumi Watanabe

Shizuoka General Hospital

Yoshinari Endo

Shizuoka General Hospital

Takahiro Suzuki

Shizuoka General Hospital

Mika Saigusa

Shizuoka General Hospital

Akito Yamamoto

Shizuoka General Hospital

\section{Satoru Morita}

Shizuoka General Hospital

\section{Yoko Sato}

Shizuoka General Hospital 
Kazuhiro Asada

Shizuoka General Hospital

\section{Research Article}

Keywords: chronic respiratory disease, elderly, pneumococcal polysaccharide vaccine, pneumococcus, pneumonia, vaccine

Posted Date: March 13th, 2021

DOl: https://doi.org/10.21203/rs.3.rs-287264/v1

License: (c) (i) This work is licensed under a Creative Commons Attribution 4.0 International License. Read Full License

Version of Record: A version of this preprint was published at BMC Pulmonary Medicine on April 16th, 2021. See the published version at https://doi.org/10.1186/s12890-021-01491-w. 


\section{Abstract}

Background: The effectiveness of the 23-valent pneumococcal polysaccharide vaccine (PPSV23) in preventing non-invasive pneumococcal pneumonia (non-IPD) has been controversial.

Methods: To evaluate the effectiveness of the PPSV23 in elderly outpatients with chronic respiratory diseases, we carried out a case-control study, including 4128 outpatients aged $\geq 65$ years, in the respiratory department.

Results: There were 320 vaccinated patients, of which 164 were diagnosed with pneumococcal pneumonia. The adjusted odds ratio was 0.39 (95\% confidence interval ( $\mathrm{Cl}), 0.17$ to 0.89 ). In the subsets consisting of age groups $\geq 70$ and $\geq 75$ years, the adjusted odds ratio $(95 \% \mathrm{Cl})$ was respectively 0.16 (0.04 to 0.67$)$ and 0.15 (0.02 to 1.12$)$.

Conclusion: This real-world study suggests that PPSV23 is effective in preventing pneumococcal pneumonia in the elderly with chronic respiratory diseases and in the older population (age $\geq 75$ years).

\section{Background}

Streptococcus pneumonia can cause pneumonia and invasive pneumococcal diseases (IPDs), which result in considerable morbidity and mortality worldwide [1, 2]. The Advisory Committee on Immunization Practices recommends the use of the 23-valent pneumococcal polysaccharide vaccine (PPSV23) or the 13-pneumococcal conjugate vaccine for all the elderly (age $\geq 65$ years) and for immunocompromised adults [3]. Although the effectiveness of the PPSV23 in preventing IPD has been reported, its effectiveness in preventing non-invasive pneumococcal pneumonia (non-IPD) has been inconsistent [46]. Some researchers have targeted both healthy individuals and patients with various diseases at nursing home residences $[5,7]$.

We hypothesized that the PPSV23 would be useful in preventing pneumococcal pneumonia in elderly outpatients with chronic respiratory diseases. This study aimed to assess the effectiveness of the PPSV23 among elderly outpatients in clinical practice.

\section{Methods}

\section{Study design and population}

This study was a retrospective case-control design. The target population was defined as outpatients aged $\geq 65$ years, with chronic respiratory diseases, treated between 2015 and 2017 in the respiratory department of Shizuoka General Hospital. From this sample, the case and control groups consisted of patients with and without pneumococcal pneumonia, respectively.

\section{Diagnosis of non-invasive pneumococcal pneumonia}


Respiratory physicians diagnosed pneumonia based on clinical findings such as fever, hypothermia, chills, cough, sputum production, pleuritic chest pain, fatigue, tachypnea, white blood cell count $>9300$, or $<4000$ cells $/ \mathrm{mm}^{3}$, and new pulmonary infiltrates on chest radiography [2]. In this study, all patients with pneumonia met these criteria. Non-IPD was diagnosed based on the positive results of urine pneumococcal antigen and sputum culture, but a negative blood culture for pneumococcus.

\section{Definitions}

The chronic respiratory diseases in this study included lung cancer, asthma, chronic obstructive pulmonary disease (COPD), interstitial pneumonia, pulmonary non-tuberculous mycobacteriosis (NTM), pulmonary tuberculosis, and others. The history of PPSV23 vaccination was obtained from medical records and declarations by patients or their families. Patients were considered vaccinated when they had received the PPSV23 within five years prior to the diagnosis of pneumonia. Patients without medical records or whose families had no knowledge of their vaccination statuses were considered unvaccinated.

\section{Statistical analysis}

The chi-squared tests for categorical variables and t-tests for continuous variables were used in comparing both groups. To evaluate the effectiveness of the PPSV23, we performed a logistic regression analysis, and then the odds ratio (OR), $95 \%$ confidence interval (Cl), and p-value (based on Wald test) were calculated. The adjusted OR was estimated by the quantile stratification method of propensity scores. The propensity score was estimated using multivariate logistic regression models with potential confounders as covariates, which included all variables of table 1 . We also made two subsets: those $\geq$ 70 years, and $\geq 75$ years, and compared their adjusted ORs with that in all patients. As a sensitivity analysis, we estimated double-robust adjusted OR in case-control studies under causal inference [8], and we confirmed whether the point estimation of OR, as mentioned above, was overestimating the effect. A $p$-value of $<0.05$ was considered to be statistically significant. Statistical analyses were performed using SAS version 9.4 (SAS Institute, Cary, NC, USA).

\section{Results}

\section{Patients background}

Between January 1, 2015, and December 31, 2017, 4218 outpatients aged $\geq 65$ years with chronic respiratory diseases visited our department. The patient flow is shown in Fig. 1. A total of 320 patients received the PPSV23, while 3898 did not. Of the 320 vaccinated patients, 6 developed pneumococcal pneumonia, compared to 158 of the 3898 unvaccinated patients.

The baseline characteristics of the case and control group on January 1, 2015, are shown in Table 1. Patients in the case group were more current-smokers, as well as had other chronic respiratory diseases, 
diabetes, chronic heart disease, and had higher use of systemic corticosteroids, than the control group. The number of patients in the case group with lung cancer was less than that of the control group.

Table 1

Characteristics of patients with and without pneumococcal pneumonia.

\begin{tabular}{|c|c|c|c|}
\hline $\begin{array}{l}\text { Variable and } \\
\text { category (reference) }\end{array}$ & $\begin{array}{l}\text { Case group } \\
(\mathrm{n}=164)\end{array}$ & $\begin{array}{l}\text { Control group } \\
(n=4,054)\end{array}$ & P-value \\
\hline Age, $y^{*}$ & $76.2 \pm 7.3$ & $75.1 \pm 6.7$ & 0.127 \\
\hline $65-69$ & $32(19.5)$ & $984(24.3)$ & \multirow[t]{4}{*}{0.153} \\
\hline $70-74$ & $44(26.8)$ & $1,098(27.1)$ & \\
\hline $75-79$ & $36(22.0)$ & $950(23.4)$ & \\
\hline $80+$ & $56(34.1)$ & $1,063(26.2)$ & \\
\hline Male (vs. female) & $113(68.9)$ & $2,525(62.3)$ & 0.100 \\
\hline Current (reference: ex-smokers) & $108(65.9)$ & $2,068(51.0)$ & 0.001 \\
\hline \multicolumn{4}{|l|}{ Chronic respiratory diseases (vs. absent) } \\
\hline Asthma & $30(18.3)$ & $685(16.9)$ & 0.671 \\
\hline COPD & $42(25.6)$ & $959(23.6)$ & 0.574 \\
\hline Lung cancer & $45(27.4)$ & $1,594(39.3)$ & 0.002 \\
\hline Interstitial pneumonia & $29(17.7)$ & $703(17.3)$ & 0.916 \\
\hline NTM & $10(6.1)$ & $449(11.1)$ & 0.054 \\
\hline Others $^{\dagger}$ & $48(29.3)$ & $746(18.4)$ & 0.001 \\
\hline Diabetes (vs. absent) & $65(39.6)$ & $1,209(29.8)$ & 0.023 \\
\hline Chronic heart disease (vs. absent) & $95(57.9)$ & $1,891(46.6)$ & 0.005 \\
\hline Chronic kidney disease (vs. absent) & $13(7.9)$ & $299(7.4)$ & 0.761 \\
\hline Systemic corticosteroid user (vs. absent) & $65(39.6)$ & $1,189(29.3)$ & 0.007 \\
\hline \multicolumn{4}{|c|}{ Values are expressed as numbers and proportions in parentheses. } \\
\hline \multicolumn{4}{|c|}{$\begin{array}{l}{ }^{\dagger} \text { Other chronic respiratory diseases included chronic pulmonary aspergillosis, old pulmonary } \\
\text { tuberculosis, sarcoidosis, and chronic cough. }\end{array}$} \\
\hline \multicolumn{4}{|c|}{ COPD: chronic obstructive pulmonary disease, NTM: non-tuberculous mycobacteriosis. } \\
\hline
\end{tabular}




\section{Effectiveness of the vaccine against pneumococcal pneumonia}

Pneumococcal pneumonia prevention crude OR $(95 \% \mathrm{Cl})$ was $0.45(0.20-1.03, \mathrm{p}=0.059)$, and the adjusted OR was 0.39 (0.17-0.89) (Fig. 2). There was a trend towards lower OR in older patients: higher OR $(95 \% \mathrm{Cl})$ in $\geq 65$ years, $0.16(0.04-0.67)$ in subset 1 ( $\geq 70$ years), and $0.15(0.02-1.12)$ in subset 2 ( $\geq$ 75 years). In the sensitivity analysis, the double-robust adjusted ORs in patients of $\geq 65, \geq 70$, and $\geq 75$ years old was respectively $0.35,0.14$ and, thus, the above adjusted ORs was conservative and not overestimating.

\section{Discussion}

This study found that the PPSV23 prevented non-IPD in older patients (age $\geq 65$ years) with chronic respiratory diseases, and could be more effective for the elderly (patients aged $\geq 70$ years). To the best of our knowledge, this is the first real-world study that assesses the effectiveness of the PPSV23 in older patients with chronic respiratory disease in a single center.

In previous studies, the effectiveness of the vaccine against pneumococcal pneumonia was controversial. In the results of a meta-analysis of 18 randomized trials [4], the PPSV23 reduced the risk of IPDs such as bacteremia, meningitis, and bacteremic pneumonia (OR [95\% Cl]: $0.26[0.15-0.46]$ ) and non-IPD (0.46 [0.25-0.84]). However, some studies reported that the PPSV23 did not reduce non-IPD [5, 6, 9]. The EVAN-65 study in community-dwelling patients [10] showed that the hazard ratios (HR) for the risk of pneumococcal pneumonia in vaccinated patients compared with non-vaccinated patients were not different (HR [95 \% Cl]: 0.61 [0.35-1.06]). Similarly, another study in elderly patients with chronic respiratory diseases showed no difference $(0.76[0.30-1.90])[11]$. These conflicting results can be due to the difficulties of accurately diagnosing pneumococcal pneumonia and the use of non-validated diagnostic tests [12]. In this study, we included non-invasive cases according to specific diagnostic criteria of pneumonia, urine pneumococcal antigen, and sputum culture, and we are convinced that these results are close to correct.

One previous case-control study suggested that $85-90 \%$ of adults aged 55 and younger achieved the prevention of invasive pneumococcal infections, but this effectiveness was reduced with increasing age, and no protection was shown in the population aged $\geq 80$ years [13-15]. These studies suggest that poorer effectiveness might be due to immunosenescence, which refers to the decline of the immune system associated with aging [16]. However, this study found that the OR was much decreased in older people, which could imply that older people can acquire antibodies with the PPSV23 vaccination, and we proposed that older people be re-vaccinated because of an anticipated decline in the effectiveness of the vaccine over time.

Almost all patients with chronic respiratory diseases in this study had risk factors for pneumonia [17]. Previously, most studies on the PPSV23 targeted nursing home residences or healthy adults, and 
controversial results have been reported on non-invasive pneumonia [7]. Our findings suggest the importance of vaccinating chronic respiratory patients in clinical practice.

There were several limitations. First, this study was a retrospective single-center study, and a complete confounding adjustment was not done. Second, we did not assess the severity of the underlying diseases. The study population was biased, consisting mainly of moderate to severe disease cases. Third, we did not regularly identify the serotype of each pneumococcal pneumonia.

In summary, the PPSV23 was useful in preventing non-IPD among the elderly with chronic respiratory diseases and in patients $\geq 85$ years.

\section{Declarations}

\section{Author contributions}

Study concept and design: TM, EN, TS; acquisition of data: TM, TS, TA, KT, ST, YT, HW, YE, TS, MS, AY, SM, KA; data analysis: TM, EN, YS; interpretation of data: TM, EN, YS, TS; first draft of the manuscript: TM; All authors reviewed and approved the final manuscript for submission.

\section{Funding}

There was no funding support.

\section{Availability of data and materials}

The datasets used and/or analysed during the current study are available from the corresponding author on reasonable request.

\section{Ethics approval and consent to participate}

This study conforms to the Ethical Principles for Medical Research Involving Human Subjects issued by the Ministry of Health, Labour and Welfare and the Ministry of Education, Culture, Sports, Science, and Technology in Japan. Following these guidelines, the Shizuoka General Hospital Research Ethical Committee determined that individual patient informed consent was not required because this study was an analysis study of existing information and patients were given the right to refuse participation by disclosure. After obtaining the approval of this committee (SGHIRB\#2017062) and publishing the disclosure document on Shizuoka General Hospital's website, the information of each individual was anonymized, and the analysis was conducted. 


\section{Consent for publication}

Not applicable.

\section{Competing Interest}

The authors have no conflicts of interest to disclose.

\section{Acknowledgments}

Not applicable.

\section{Author Details}

${ }^{1}$ Department of Respiratory Medicine, Shizuoka General Hospital, 4-27-1, Shizuoka, Japan. ${ }^{2}$ Division of Clinical Biostatistics, Research Support Center, Shizuoka General Hospital, Shizuoka, Japan.

\section{References}

1. Said MA, Johnson HL, Nonyane BAS, Deloria-Knoll M, O'Brien KL, for the AAPBST. Estimating the Burden of Pneumococcal Pneumonia among Adults: A Systematic Review and Meta-Analysis of Diagnostic Techniques. PLOS ONE. 2013;8:e60273.

2. Mandell LA, Wunderink RG, Anzueto A, Bartlett JG, Campbell GD, Dean NC, et al. Infectious Diseases Society of America/American Thoracic Society consensus guidelines on the management of community-acquired pneumonia in adults. Clinical infectious diseases: an official publication of the Infectious Diseases Society of America. 2007;44 Suppl 2:S27-72.

3. Tomczyk S, Bennett NM, Stoecker C, Gierke R, Moore MR, Whitney CG, et al. Use of 13-valent pneumococcal conjugate vaccine and 23-valent pneumococcal polysaccharide vaccine among adults aged $\geq 65$ years: recommendations of the Advisory Committee on Immunization Practices (ACIP). MMWR Morbidity and mortality weekly report. 2014;63:822-5.

4. Moberley S, Holden J, Tatham DP, Andrews RM. Vaccines for preventing pneumococcal infection in adults. Cochrane Database Syst Rev. 2013:Cd000422.

5. Jackson LA, Neuzil KM, Yu O, Benson P, Barlow WE, Adams AL, et al. Effectiveness of pneumococcal polysaccharide vaccine in older adults. The New England journal of medicine. 2003;348:1747-55.

6. Musher DM, Rueda-Jaimes AM, Graviss EA, Rodriguez-Barradas MC. Effect of pneumococcal vaccination: a comparison of vaccination rates in patients with bacteremic and nonbacteremic pneumococcal pneumonia. Clinical infectious diseases: an official publication of the Infectious Diseases Society of America. 2006;43:1004-8. 
7. Maruyama T, Taguchi O, Niederman MS, Morser J, Kobayashi H, Kobayashi T, et al. Efficacy of 23valent pneumococcal vaccine in preventing pneumonia and improving survival in nursing home residents: double blind, randomised and placebo controlled trial. BMJ (Clinical research ed). 2010;340:c1004.

8. Tchetgen Tchetgen EJ, Rotnitzky A. Double-robust estimation of an exposure-outcome odds ratio adjusting for confounding in cohort and case-control studies. Stat Med. 2011;30:335-47.

9. Huss A, Scott P, Stuck AE, Trotter C, Egger M. Efficacy of pneumococcal vaccination in adults: a metaanalysis. CMAJ: Canadian Medical Association journal = journal de l'Association medicale canadienne. 2009;180:48-58.

10. Vila-Córcoles A, Ochoa-Gondar O, Hospital I, Ansa X, Vilanova A, Rodríguez T, et al. Protective effects of the 23-valent pneumococcal polysaccharide vaccine in the elderly population: the EVAN-65 study. Clinical infectious diseases: an official publication of the Infectious Diseases Society of America. 2006;43:860-8.

11. Ochoa-Gondar O, Vila-Corcoles A, Ansa X, Rodriguez-Blanco T, Salsench E, de Diego C, et al. Effectiveness of pneumococcal vaccination in older adults with chronic respiratory diseases: results of the EVAN-65 study. Vaccine. 2008;26:1955-62.

12. Musher DM. How effective is vaccination in preventing pneumococcal disease? Infect Dis Clin North Am. 2013;27:229-41.

13. Shapiro ED, Berg AT, Austrian R, Schroeder D, Parcells V, Margolis A, et al. The Protective Efficacy of Polyvalent Pneumococcal Polysaccharide Vaccine. New England Journal of Medicine. 1991;325:1453-60.

14. Suzuki M, Dhoubhadel BG, Ishifuji T, Yasunami M, Yaegashi M, Asoh N, et al. Serotype-specific effectiveness of 23-valent pneumococcal polysaccharide vaccine against pneumococcal pneumonia in adults aged 65 years or older: a multicentre, prospective, test-negative design study. The Lancet Infectious Diseases. 2017;17:313-21.

15. Kolditz M, Schmitt J, Pletz MW, Tesch F. Impact of pneumococcal polysaccharide vaccine on incidence and mortality after pneumonia in adults aged $>/=60$ years-a population-based retrospective cohort study. Clin Microbiol Infect. 2018;24:500-4.

16. Pera A, Campos C, Lopez N, Hassouneh F, Alonso C, Tarazona R, et al. Immunosenescence: Implications for response to infection and vaccination in older people. Maturitas. 2015;82:50-5.

17. Ishiguro T, Takayanagi N, Yamaguchi S, Yamakawa H, Nakamoto K, Takaku Y, et al. Etiology and factors contributing to the severity and mortality of community-acquired pneumonia. Intern Med. 2013;52:317-24.

\section{Figures}




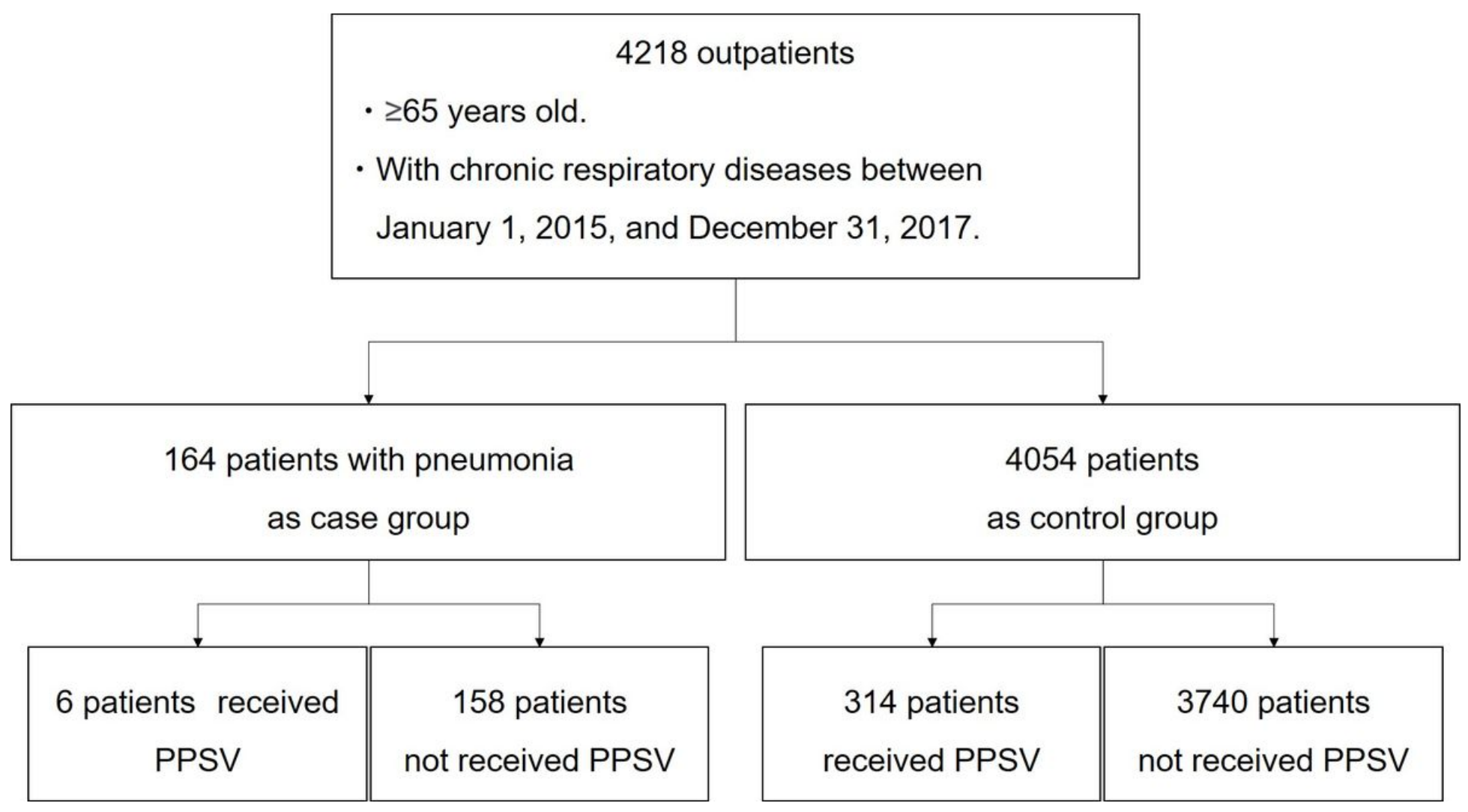

Figure 1

Patient flow. PPSV23: 23-valent pneumococcal polysaccharide vaccine

\begin{tabular}{|c|c|c|}
\hline Group & $\begin{array}{c}\text { Adjusted } \\
\text { OR }\end{array}$ & $95 \% \mathrm{Cl}$ \\
\hline
\end{tabular}

Subset $1(\geq 70) \quad 0.16 \quad 0.04$ to $0.67 \quad 0.010$

Subset $2(\geq 75) \quad 0.15 \quad 0.02$ to $1.12 \quad 0.065$

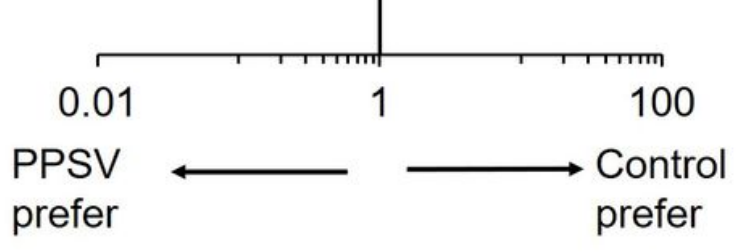

Figure 2 
Effectiveness of vaccines in pneumococcal pneumonia in age-related subsets. OR: odds ratio, Cl:

confidence interval, PPSV23: 23-valent pneumococcal polysaccharide vaccine. The lower adjusted ORs in subsets of older patients were estimated, suggesting that the PPSV23 can be more effective for older patients. The adjusted OR was adjusted using the quantile category of propensity scores. 